Characterization of GaSb-based heterostructures by scanning electron microscope cathodoluminescence and scanning tunnelling microscope

This article has been downloaded from IOPscience. Please scroll down to see the full text article.

2004 J. Phys.: Condens. Matter 16 S251

(http://iopscience.iop.org/0953-8984/16/2/030)

View the table of contents for this issue, or go to the journal homepage for more

Download details:

IP Address: 147.96.14.16

The article was downloaded on 21/02/2013 at 13:21

Please note that terms and conditions apply. 


\title{
Characterization of GaSb-based heterostructures by scanning electron microscope cathodoluminescence and scanning tunnelling microscope
}

\author{
J Storgårds ${ }^{1}$, B Méndez ${ }^{1,3}$, J Piqueras $^{1}$, M Chenot ${ }^{2}$, F Dimroth $^{2}$ and \\ A W Bett ${ }^{2}$ \\ ${ }^{1}$ Departamento de Física de Materiales, Facultad de Ciencias Físicas, Universidad Complutense \\ de Madrid, 28040 Madrid, Spain \\ ${ }^{2}$ Fraunhofer Institute for Solar Energy Systems, 79110 Freiburg, Germany \\ E-mail: bianchi@fis.ucm.es
}

Received 31 July 2003

Published 22 December 2003

Online at stacks.iop.org/JPhysCM/16/S251 (DOI: 10.1088/0953-8984/16/2/030)

\begin{abstract}
The luminescence of GaSb and AlGaSb layers grown on GaAs substrates by metal organic vapour phase epitaxy has been studied by means of cathodoluminescence (CL) using a scanning electron microscope. CL planeview analysis reveals a distribution of defects, as misfit dislocations, in some of the structures. The luminescence bands observed in the GaSb layers are related to near band edge recombination and to an excess of Ga. In the case of $\mathrm{AlGaSb} / \mathrm{GaSb}$ heterostructure emission bands related to the ternary compound and to donor-acceptor recombination are detected. In addition, with the aid of a scanning tunnelling microscope (STM), local electronic properties, such as conductance and surface energy gap, were studied in sample cross-sections. The results obtained from imaging and spectroscopy modes of STM enabled us to image the single $\mathrm{AlGaSb}$ layer.
\end{abstract}

(Some figures in this article are in colour only in the electronic version)

\section{Introduction}

Gallium antimonide and its related ternary and quaternary compounds are increasingly utilized for long wavelength lasers, photodetectors and thermophotovoltaic applications [1]. These compounds emerge as a novel family of materials for developing thermophotovoltaic devices, due to the fact that their bandgap extends beyond the limit of traditional silicon photovoltaic cells [2]. For this reason, it is necessary to improve the quality of the GaSbbased microstructures and to obtain a deep knowledge of their physical properties. Although

3 Author to whom any correspondence should be addressed. 


\begin{tabular}{lll} 
Table 1. & Structure of the samples investigated \\
\hline Sample & Layer & Thickness $(\mathrm{nm})$ \\
\hline $\mathrm{G} 1$ & $\mathrm{GaSb}$ & 800 \\
& $\mathrm{GaSb}$ buffer & 200 \\
$\mathrm{G} 2$ & $\mathrm{Al}_{0.2} \mathrm{Ga}_{0.8} \mathrm{Sb}$ & 50 \\
& $\mathrm{AlGaSb}$ & 50 \\
& $\mathrm{GaSb}$ & 1112,4 \\
\hline
\end{tabular}

epitaxial layers of both binary and ternary compounds have been grown by the main growth techniques $[3,4]$ relatively little work has been carried out on problems of defect structure and defect electronic levels and their relationship to the growth conditions.

In this work, a GaSb layer and a ternary $\mathrm{AlGaSb} / \mathrm{GaSb}$ heterostructure were grown on GaAs substrates using metal organic vapour phase epitaxy (MOVPE). The structures were investigated by cathodoluminescence (CL) in a scanning electron microscope (SEM) as well as by scanning tunnelling microscope (STM) based techniques. CL enables the study of problems related to the nature and distribution of defects, electronic levels or recombination properties with high spatial resolution. The STM is mounted in the chamber of the SEM which enables us to perform correlative microscopy of sample features with a spatial resolution which varies from the micron range to tens of nanometres. In order to study the local electronic properties of the single layers the sample cross-section was studied by scanning tunnelling microscopy and spectroscopy.

\section{Experimental details}

The MOVPE growth of GaSb on GaAs substrate was performed on a production-size AIX2600G3 MOVPE reactor with an $8 \times 4$ inch configuration. 2 inch GaAs wafers were placed into the middle of a 4 inch recess. Triethylgallium (TEGa), trimethylantimony (TMSb), and dimethylethylamine alane (DMEAA) were used as source materials. The growth temperature was $575^{\circ} \mathrm{C}$ and the growth rate for GaSb was $0.9 \mu \mathrm{m} \mathrm{h}^{-1}$ at a V/III ratio of 2 . The reactor pressure was 100 mbar. The carrier-gas flow of Pd-purified $\mathrm{H}_{2}$ was in the range of 28 slpm. (100) semi-insulating GaAs wafers with $6^{\circ}$ misorientation towards (111)A were used. The substrates were directly loaded to the MOVPE reactor. The structure of the samples is shown in table 1. The samples were observed in the CL mode of operation in a Hitachi S2500 SEM at a temperature of $90 \mathrm{~K}$ and at an accelerating voltage of $20 \mathrm{kV}$. For the detection of near-infrared light a cooled ADC germanium detector was employed. The sub-microscopic measurements were performed in a combined SEM-STM system based on a Leica 440 SEM operating under a vacuum of $1 \times 10^{-6}$ Torr. The small size of the STM enabled it to be mounted on the SEM specimen holder. Mechanically sharpened Pt-Ir wires were used as probe tips for tunnelling experiments. The STM was used in the conventional constant-current mode and in the current imaging tunnelling mode (CITS). CITS provides real space imaging of surface electronic states by recording $I-V$ characteristic curves at fixed tip-sample separation at every pixel within an image [5]. The STS mode provides information on surface electronic states by recording $I-V$ curves at fixed tip-sample separation [6]. The $I-V$ curves obtained at every point depend, through the tunnelling current, on both tip-sample separation and applied bias voltage. Most of this dependence can be removed [6] by plotting the ratio of differential to total conductance, $(\mathrm{d} I / \mathrm{d} V) /(I / V)$, which provides a rather direct measure of the surface density of states [7]. 

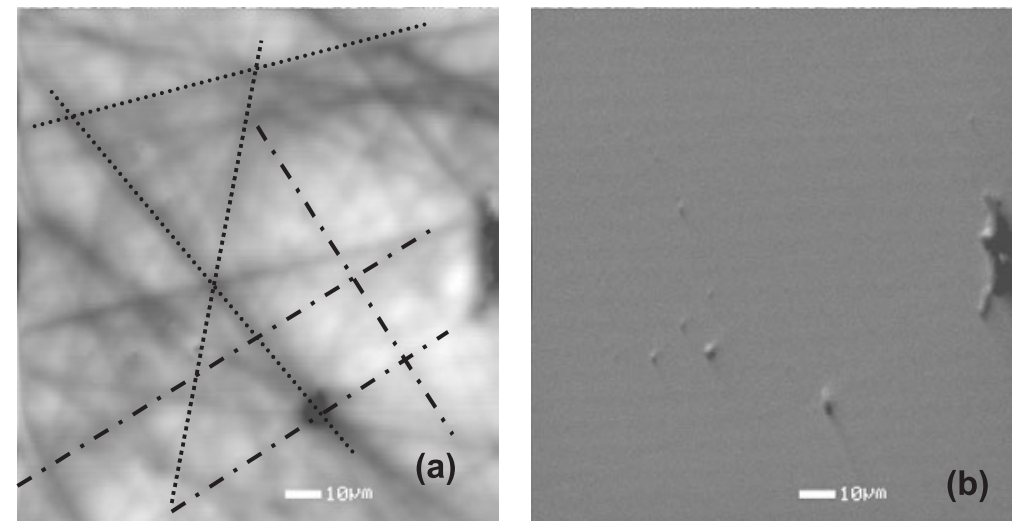

Figure 1. (a) $\mathrm{CL}$ and (b) SE images of sample G1. The accelerating voltage was $15 \mathrm{kV}$ and the temperature $90 \mathrm{~K}$. Lines marked with dots or dashes indicate $60^{\circ}$ and $90^{\circ}$ net lines respectively.

\section{Results and discussion}

\subsection{Cathodoluminescence}

Figure 1 shows the CL plane-view image of sample G1 and the corresponding secondary electron (SE) image. The CL image reveals a network of dark lines. This contrast is attributed to misfit dislocations, which appear in mismatched heterostructures. GaSb and GaAs exhibit a $7.8 \%$ lattice constant mismatch which results in a high density of dislocations in the GaSb epilayer (see, for example, [8, 9]). Kim et al [10] investigated the initial nucleation of GaSb on (001) GaAs by MOCVD using TEM and showed that the GaSb/GaAs misfit strain is very sensitive to the growth temperature. For growth at $520^{\circ} \mathrm{C}$, the misfit strain was mainly accommodated by $90^{\circ}$ dislocations, whereas for growth at $560^{\circ} \mathrm{C}$, the misfit strain was accommodated by $60^{\circ}$ dislocations, which caused a tilt of $\mathrm{GaSb}$ islands with respect to the GaAs substrate. The CL images of the present work reveal two sets of lines; one set consists of $90^{\circ}$ crossing lines, while in the other set the lines cross at an angle of $60^{\circ}$. The CL analysis of the whole sample shows that the net of $60^{\circ}$ is dominant. This is in agreement with what is expected at a growth temperature of $575^{\circ} \mathrm{C}$. In the CL image of figure 1 some of the lines forming $60^{\circ}$ angles have been marked with dots. A rough measure of the density of lines in the CL images gives values between $7 \times 10^{4}$ and $1.4 \times 10^{5} \mathrm{~cm}^{-2}$. These figures are in agreement with the typical dislocation density in available GaAs substrates. The background exhibits an uniform luminescence distribution.

The CL spectrum recorded at a temperature of $90 \mathrm{~K}$ with an accelerating voltage of $15 \mathrm{kV}$ is shown in figure 2. In the figure, the points correspond to experimental data and the continuous curve is the sum of two deconvoluted Gaussian bands that best fit the experimental data. The resolved emissions correspond to the near band edge (NBE) of GaSb at $798 \mathrm{meV}$ and to a defect-related emission at $755 \mathrm{meV}$. Our previous CL study of bulk GaSb obtained by the Bridgmann method [11] revealed two dominant emission bands, the NBE band at $796 \mathrm{meV}$ and the so-called band $\mathrm{A}$ at $777 \mathrm{meV}$, associated with a native defect $\left(\mathrm{Ga}_{\mathrm{Sb}}-\mathrm{V}_{\mathrm{Ga}}\right)$. Besides these well-known emissions, another defect-related emission band centred at $756 \mathrm{meV}$ (band B) was detected, which corresponds to the defect band observed in the spectrum of figure 2. This band appears under gallium atmosphere thermal treatment and we have proposed it to be related to an antisite $\mathrm{Ga}$ defect. The fact that the $777 \mathrm{meV}$ native defect band usually present does not appear in the GaSb layer indicates the good quality of the sample. This 


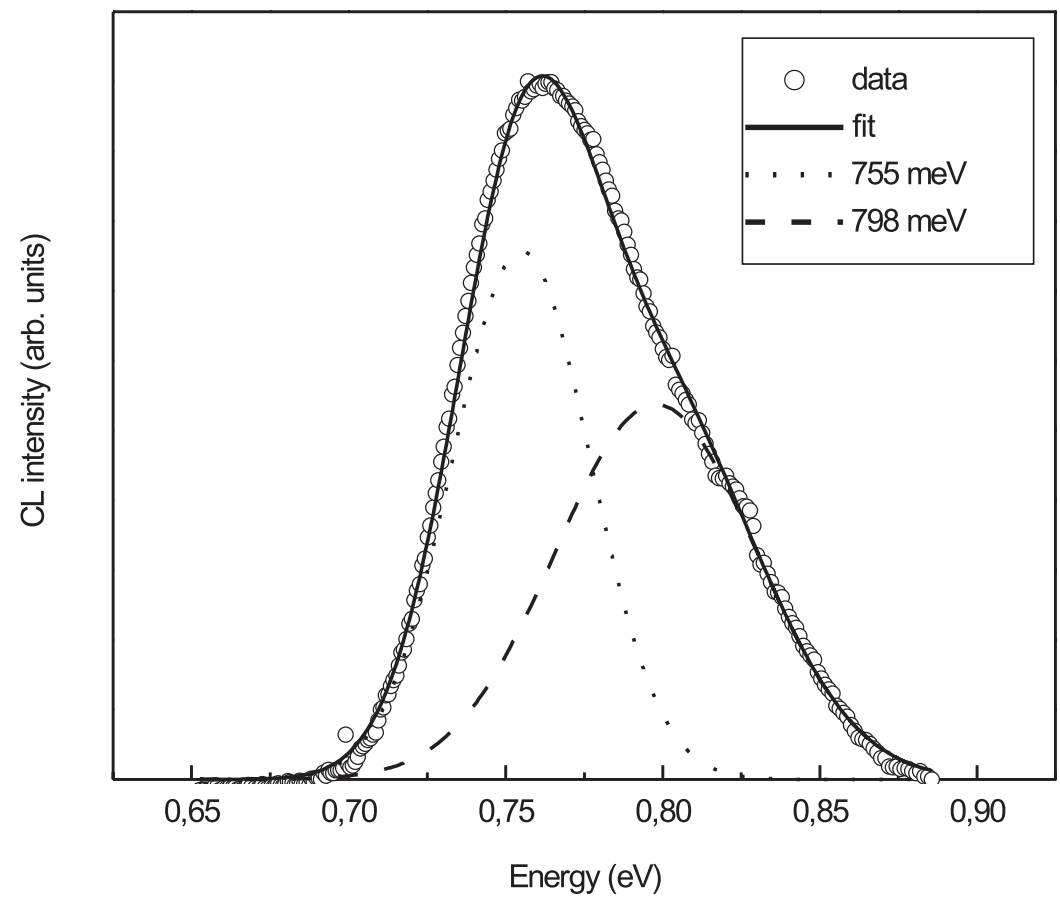

Figure 2. CL spectrum at $15 \mathrm{kV}$ and at $90 \mathrm{~K}$ from sample $\mathrm{G} 1$. The experimental data (points) were fitted to Gaussian curves (dotted and dashed curves). In the inset the maximum energy of the bands is shown.

can be explained by the Sb-rich condition in the growth procedure. The excess of Sb would hamper the formation of native lattice defects, such as the above-mentioned antisite-vacancy complex. This fact has been previously reported for LPE GaSb layers grown from Sb-rich solutions [12], in which a correlation between a decrease in the PL intensity of the band A with a lower concentration of native defects was found. Previous PL measurements in undoped and Si-doped GaSb [13] show that the band A also decreases if $\mathrm{Si}$ is used as dopant and that there is always a very shallow level of Si during MOVPE growth. On the other hand, the defect band $\mathrm{B}$ appears in the CL spectra of the GaSb layer. Although the centre or complex responsible for this emission is not unambiguously identified, we assume that the specific experimental conditions (in particular the growth temperature and the III-V ratio) inhibit the formation of the antisite-vacancy complex and possibly only the antisite defect (Ga atoms on $\mathrm{Sb}$ sites) remains.

The peak positions of the luminescence bands observed in the GaSb layer appear slightly shifted towards higher energies relative to the positions obtained from bulk material in our previous investigations. This agrees with previous photoluminescence (PL) studies on GaSb layers. Chidley et al [14] carried out a PL study of GaSb grown by MOVPE on various substrates and they found shifts of up to $4 \mathrm{meV}$ in PL spectra for material grown on GaAs. This was explained by the presence of strain in the epitaxial layer because of differential thermal expansion. In our case the layer thickness is high enough to release the internal strain originating at the interface by the nucleation of misfit dislocations as the CL images reveal.

Figure 3 shows CL and SE plane-view images of sample G2. The SE image (figure 3(a)) shows a background of hillocks of which some are larger than others. The spatial distribution of these features seems to be random over the sample without any preferential orientation and only 

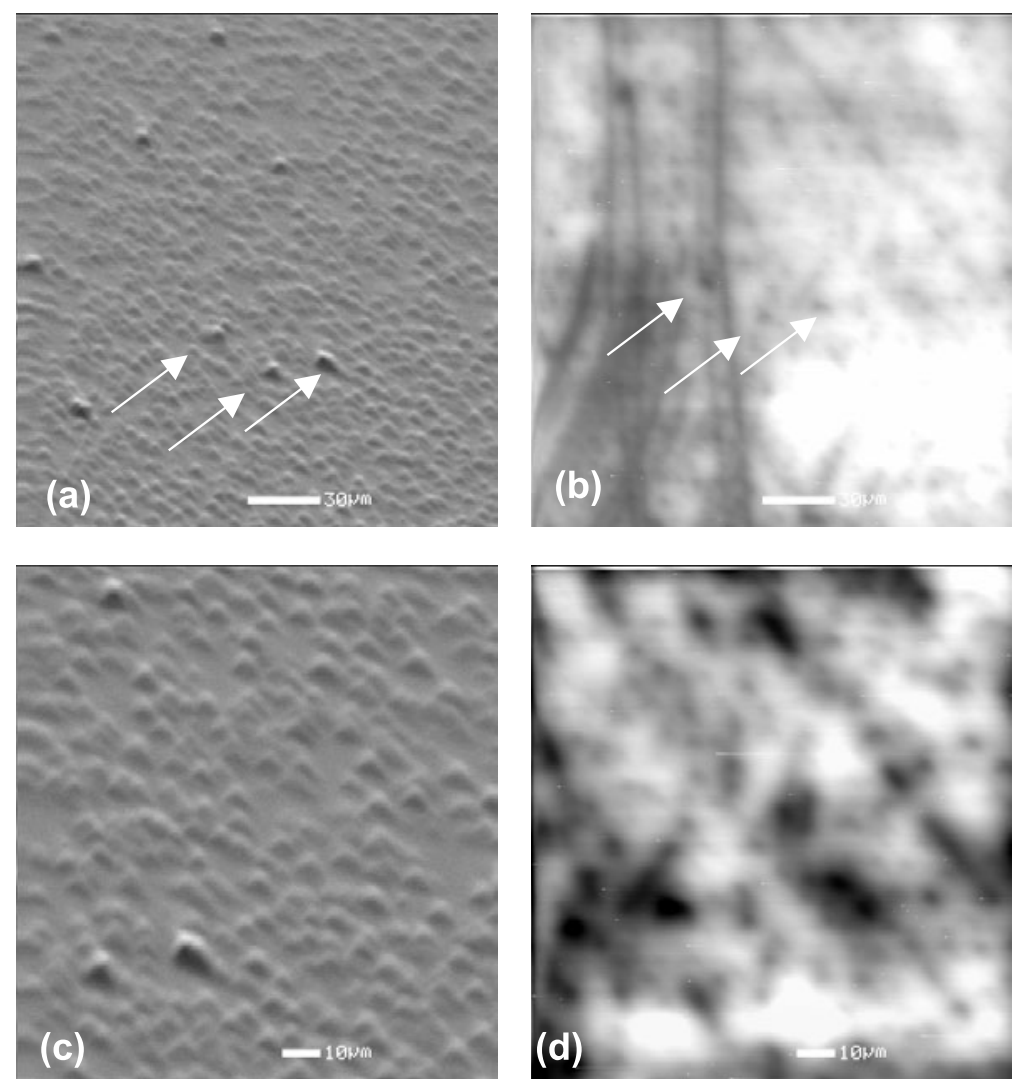

Figure 3. (a) SE image and (b) CL plane-view images of the $\mathrm{AlGaSb} / \mathrm{GaSb}$ heterojunction grown on GaAs, sample G2. The accelerating voltage was $20 \mathrm{kV}$ and the temperature $90 \mathrm{~K}$. Some hillocks in the SE image are marked with arrows as well as the corresponding dark dots in the CL image. (c) SE and (d) CL images of the same region at higher magnification.

the higher and bigger hillocks, marked with arrows, show a dark contrast in the corresponding CL image (figure 3(b)). Other features in the CL image are dark lines and small dark spots on a bright uniform background. Some of the spots are arranged following a straight line. At higher magnification, it can be clearly seen that neither preferential distribution nor lines of hillocks are inferred from the SE (figure 3(c)), as comparison with the CL image (figure 3(d)) shows. The CL dark spot contrast has been associated with emergence points of dislocations in many III-V compounds and other semiconductors. The size of the dots is between 5 and $10 \mu \mathrm{m}$ and the density estimated from the CL image is about $3 \times 10^{5} \mathrm{~cm}^{-2}$. However, the hillock density measured from the SE images is one order of magnitude higher than the dislocation density.

Figure 4 shows the CL spectrum from sample G2 recorded with an acceleration voltage of $25 \mathrm{kV}$. We have used this energy in order to reach the AlGaSb layer. The analysis of experimental data by deconvolution in Gaussian bands reveals three emissions: a defect-related emission at $754 \mathrm{meV}$, the NBE GaSb emission at $803 \mathrm{meV}$ and a new rather weak emission at $840 \mathrm{meV}$. The two first bands originate in the GaSb layers and have been discussed above. In particular, as the thickness of the GaSb buffer layer is only $200 \mathrm{~nm}$ a shift in NBE position is detected. The $840 \mathrm{meV}$ band does not appear at lower acceleration voltages at which the ternary layer is not reached by the electron beam. Therefore, the presence of this above GaSb 


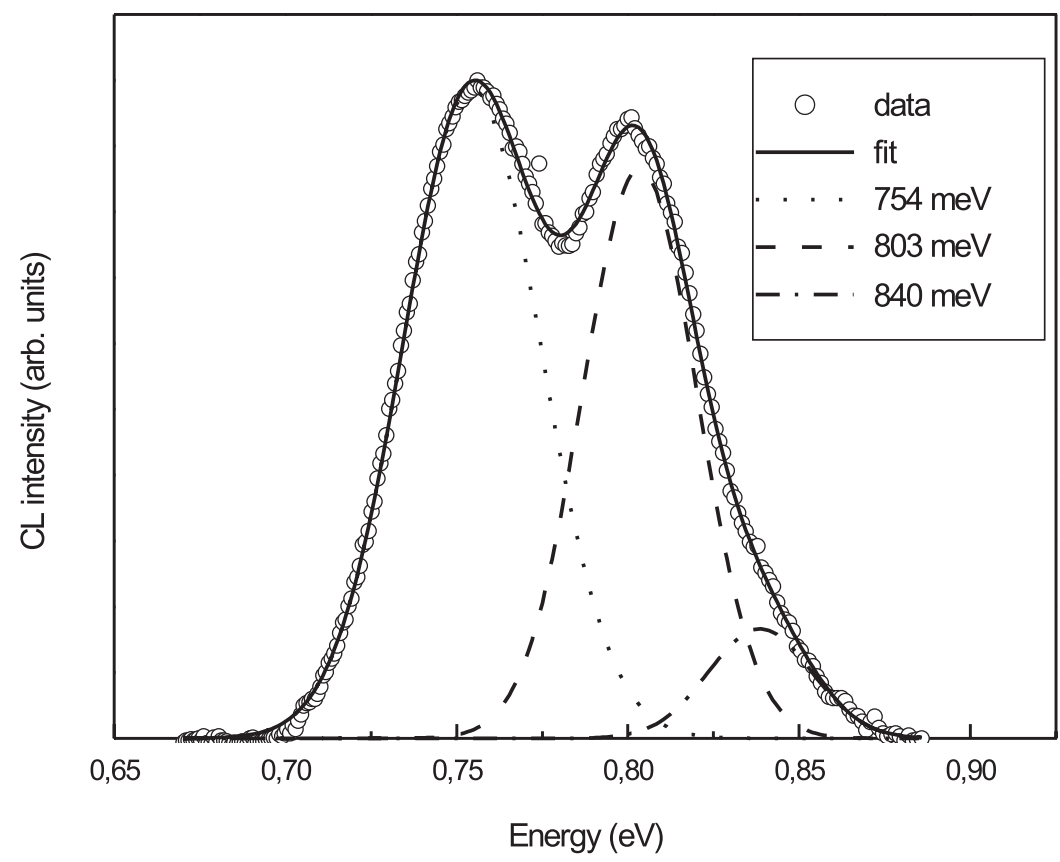

Figure 4. $\mathrm{CL}$ spectrum at $25 \mathrm{kV}$ and at $90 \mathrm{~K}$ from sample $\mathrm{G} 2$. The experimental data (points) were fitted to Gaussian curves (continuous and dashed curves). In the inset the maximum energy of the bands is shown.

gap energy emission in the spectra recorded at $25 \mathrm{kV}$ is associated with the AlGaSb layer. Kitamura et al [15] have interpreted PL measurements of AlGaSb by taking into account one shallow acceptor level and two native defect levels in the energy band diagram. The native defects introduce two levels, which are about $90 \mathrm{meV}$ below the first minimum and about $160 \mathrm{meV}$ below the second minimum of the conduction band. On the other hand, the shallow acceptor level related to the native complex defect $\left(\mathrm{V}_{\mathrm{Ga}}-\mathrm{Ga}_{\mathrm{Sb}}\right)$ in $\mathrm{GaSb}$ and also in $\mathrm{AlGaSb}$ is located at about $30 \mathrm{meV}$ above the valence band [12]. The present results point out that the possible mechanism for the $840 \mathrm{meV}$ band is a transition from the second donor native $\mathrm{Al}$ related level, as proposed in [15], to the shallow acceptor level. From these data, a band gap of about $1.03 \mathrm{eV}$ is inferred for the AlGaSb layer. This result agrees with the corresponding band gap value for an $\mathrm{Al}$ fraction of $x=0.2$.

\subsection{Scanning tunnelling}

In order to obtain a further characterization of the $\mathrm{GaSb}$ and $\mathrm{AlGaSb}$ layers grown on GaAs, we have studied local electronic properties at a sub-micron scale with the aid of an STM incorporated into the SEM chamber, which enables us to resolve the individual layers. Figure 5(a) shows a constant current tunnelling image of the cross-section of sample G1. The surface appears rather flat and homogeneous. In the region shown in the image a terrace is clearly revealed, which corresponds to the substrate material. This was confirmed from $I-V$ curves recorded at fixed tip-sample separation on different locations on the surface. From these curves the ratio of differential to total conductance $(\mathrm{d} I / \mathrm{d} V) /(I / V)$ is plotted; this provides a rather direct measure of the surface density of states. Figures 5(b) and (c) show the differential conductance curves from points labelled as 1 and 2 respectively on the surface 
$317.8 \mathrm{~nm}$
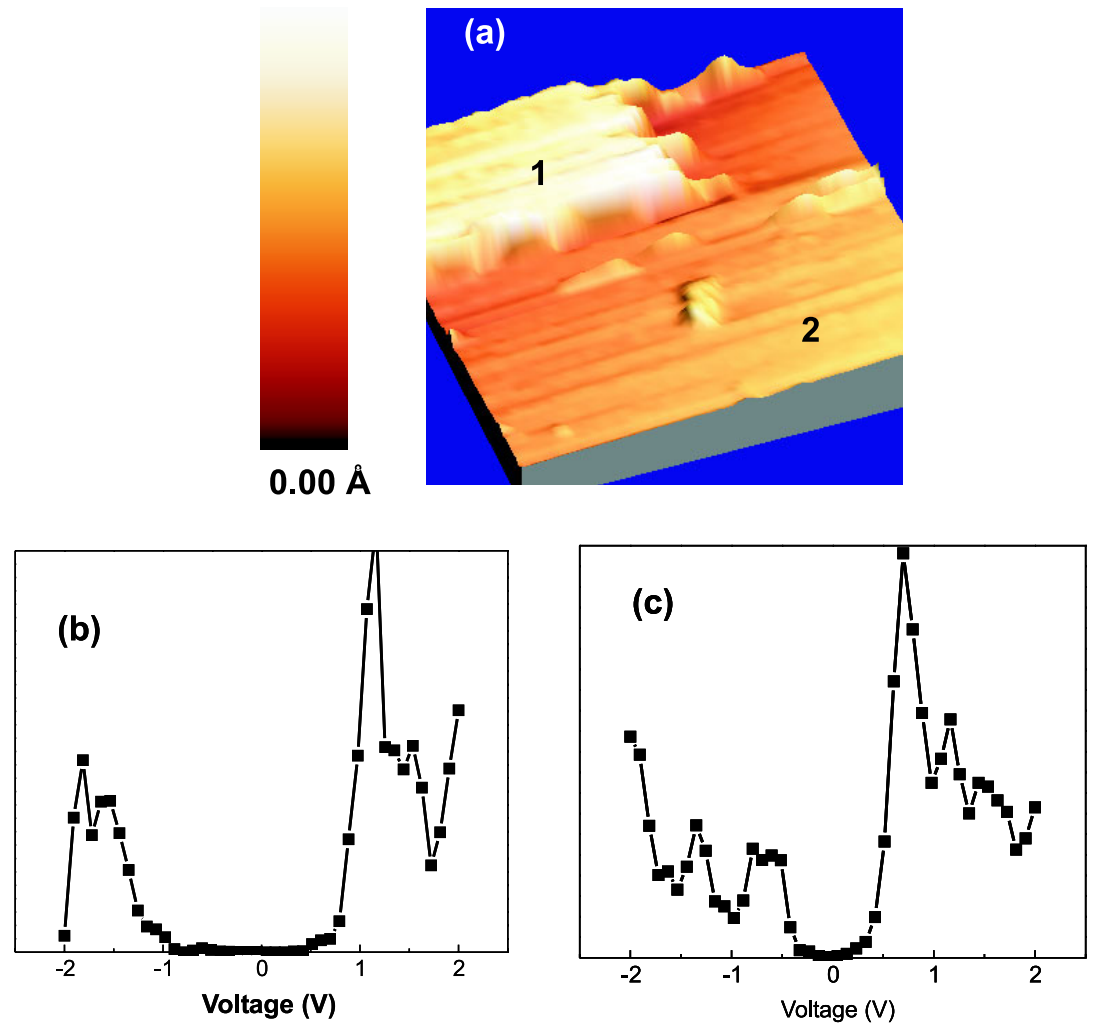

Figure 5. (a) Constant current tunnelling image of the cross-section of sample G1. The size is $2 \mu \mathrm{m} \times 2 \mu \mathrm{m}$. The tunnel current was $0.5 \mathrm{nA}$. (b) and (c) Differential conductance curves from points labelled as 1 and 2 respectively in (a).

shown in figure 5(a). From these curves, it is possible to measure the surface band gap, which is $1.3 \mathrm{eV}$ at position 1 and about $0.6 \mathrm{eV}$ at position 2. The first value is close to the bulk value of GaAs and the second one applies to GaSb. It is to be pointed out that the surface gap value could not correspond exactly to the bulk band gap because of the presence of surface states. Moreover, the effect of the tip-sample interaction cannot be readily predicted nor can it be measured using conventional STS techniques.

The constant current tunnelling image of the cross-section of sample G2 is shown in figure 6(a). The bottom part of the figure corresponds to the upper GaSb layer and the top of the image corresponds to the GaAs substrate. Differential conductance curves were obtained from selected points on the surface and figures 6(b) and (c) show representative results obtained in the regions marked 1 and 2 respectively. From curve 1, a surface band gap value of $1.0 \mathrm{eV}$ is measured. This result is in agreement with the AlGaSb band gap obtained from the CL spectra of sample G2. The curve from region 2 leads to a surface band gap of $0.4 \mathrm{~V}$ and hence it would correspond to a GaSb layer [16]. Furthermore, CITS images from this area were recorded, which enable us to detect with high spatial resolution local conductance variations. Figure 7 shows some of the CITS images obtained at different voltages. A bright stripe of about $100 \mathrm{~nm}$ which corresponds to the AlGaSb layer is revealed in the image at $+2 \mathrm{~V}$. The stripe width has 


\section{$228.67 \mathrm{~nm}$}
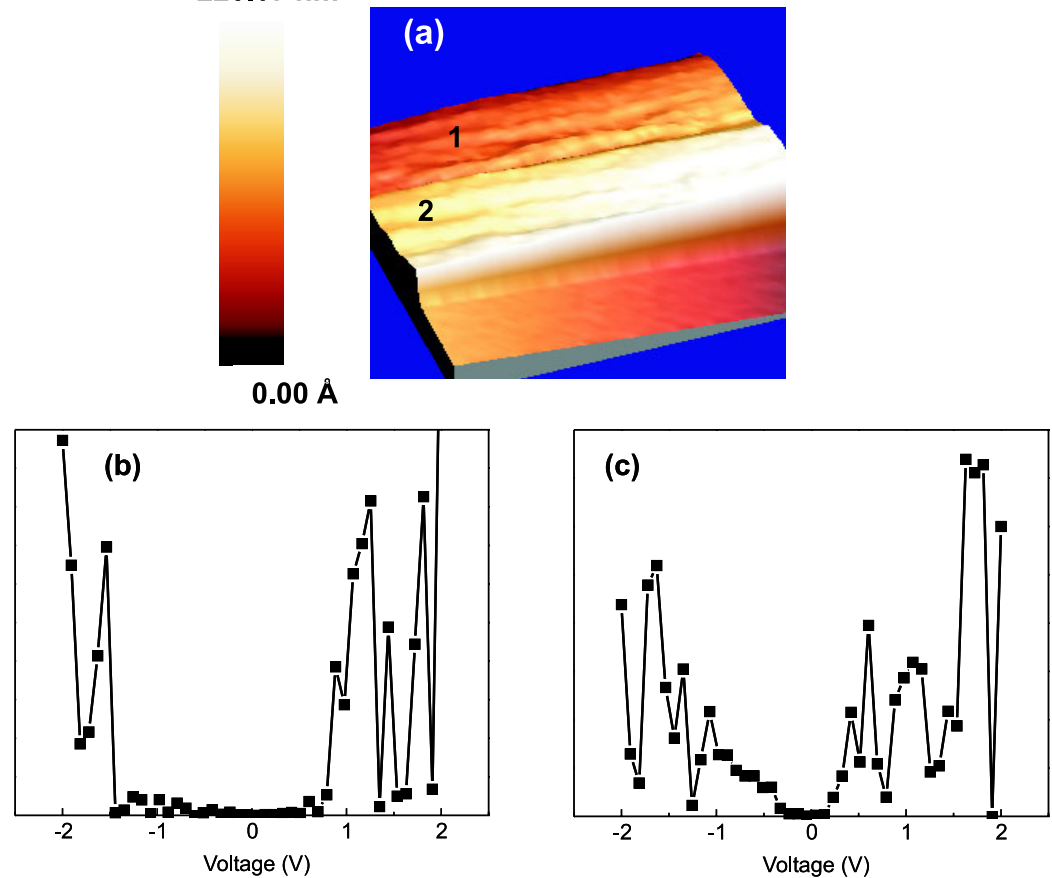

Figure 6. (a) Constant current tunnelling image of the cross-section of sample G2. The size is $2.8 \mu \mathrm{m} \times 2.8 \mu \mathrm{m}$. The tunnel current was $0.5 \mathrm{nA}$. (b) and (c) Differential conductance curves from points labelled as 1 and 2 respectively in (a).

been measured directly from the line profile shown in figure 7(e). CITS results show a bright contrast from the AlGaSb layer at voltages higher than $1.1 \mathrm{~V}$, while at low voltages the layer appears dark compared with GaSb. The contrast appears for voltages below and above the band gap. The changes in the conductance curves and the inversion contrast at $1.1 \mathrm{~V}$ revealed in CITS images support the identification of the AlGaSb layer in the cross-section image.

\section{Conclusions}

The defect structure of GaSb and AlGaSb layers grown on GaAs by MOVPE has been studied by means of CL in the SEM. CL images from GaSb layers reveal a net of dark lines forming angles of $60^{\circ}$ or $90^{\circ}$ related to misfit dislocations. In AlGaSb/GaSb layers, CL images show dark spots associated with emergence points of dislocations, which often appear arranged along lines. Although the usual native defect band at $777 \mathrm{meV}$ in $\mathrm{GaSb}$ is absent in the CL spectra, another defect-related band at $755 \mathrm{meV}$ is detected in both samples. The CL results suggest that this emission band is related to a Ga antisite defect. In the AlGaSb/GaSb sample a luminescence band at $840 \mathrm{meV}$ is resolved, which would result from radiative transitions between native defect levels in AlGaSb providing a gap value of $1.03 \mathrm{eV}$. In addition, STM analysis of sample cross-sections has been performed. The surface energy gap values obtained from differential conductance curves in the cross-section of the samples were in accordance with the nominal values for GaAs, GaSb and AlGaSb. The individual $100 \mathrm{~nm} \mathrm{AlGaSb}$ layer is revealed through the CITS images. 

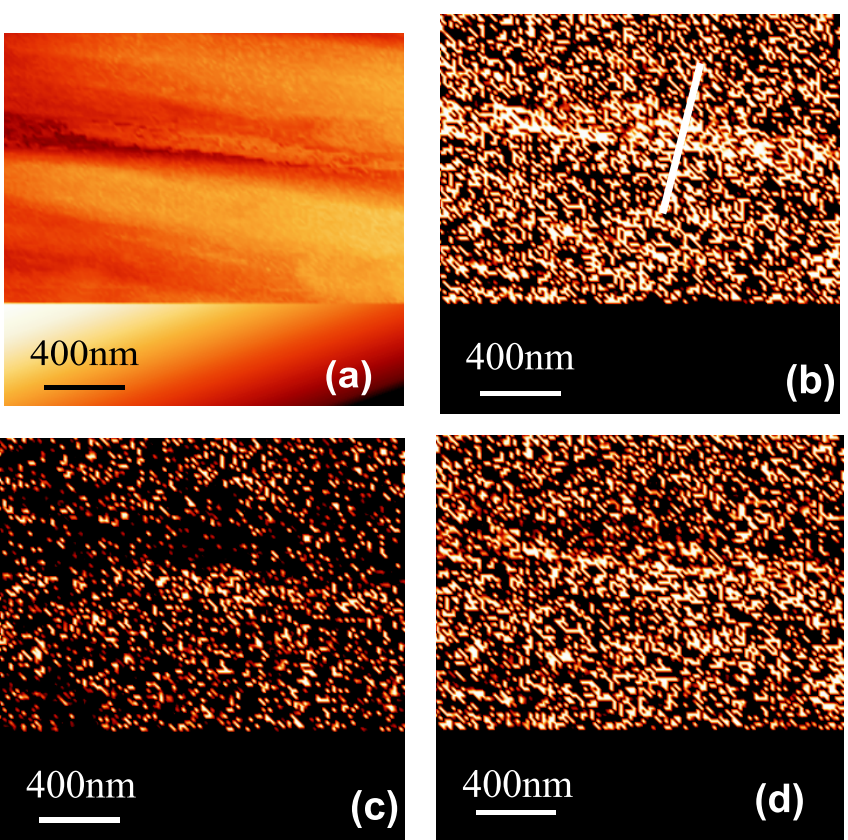

Line profile

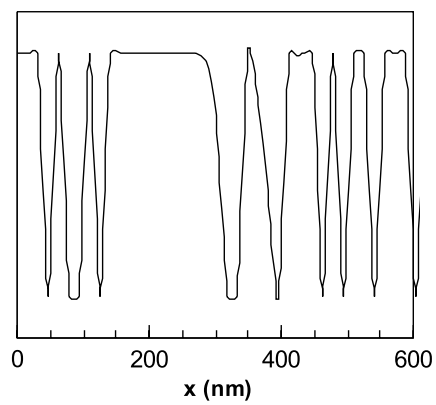

Figure 7. (a) STM constant current image of the cross-section of sample G2 and $0.4 \mathrm{nA}$ tunnelling current. Corresponding CITS images acquired at (b) $-1.91 \mathrm{~V},(\mathrm{c})+0.51 \mathrm{~V}$, and (d) $+1.16 \mathrm{~V}$ sample voltages. (e) Profile from the white line drawn in (b).

\section{Acknowledgments}

This work was carried out in the framework of a European network project (HPRN-CT-200100199). Partial support of MCYT (MAT2000-2119) is acknowledged.

\section{References}

[1] Dutta P S, Bhat H L and Kumar V 1997 J. Appl. Phys. 815

[2] Couts T J, Benner J P and Allman C S (ed) 1998 Proc. 4th NREL Conf. on Thermophotovoltaic Generation of Electricity (Denver, CO) (AIP Conf. Proc. vol 460) (New York: American Institute of Physics)

[3] Qian W, Skowronski M, Kaspi R, de Graef M and Dravid V P 1997 J. Appl. Phys. 817268

[4] Yi S S, Hansen D M, Inoki C K, Harris D L, Kuan T S and Kuech T F 2000 Appl. Phys. Lett. 77842

[5] Feenstra R M 1994 Surf. Sci. 299/300 965

[6] Stroscio J A, Feenstra R M and Fein A P 1986 Phys. Rev. Lett. 572579 
[7] Feenstra R M, Stroscio J A and Fein A P 1987 Surf. Sci. 181295

[8] Rocher A 1971 Solid State Phenom. 19/20 563

[9] Rocher A, Kang J M and Ponchet A 1992 MRS Symp. Proc. 23891

[10] Kim J, Seong T, Mason N J and Walker P J 1998 J. Electron. Mater. 27466

[11] Méndez B, Dutta P S, Piqueras J and Dieguez E 1995 Appl. Phys. Lett. 672648

[12] Jiang W J, Sun Y M and Wu M C 1995 J. Appl. Phys. 771725

[13] Agert C, Galdkov P and Bett A W 2002 Semicond. Sci. Technol. 1739

[14] Chidley E T R, Haywood S K, Henriques A B, Mason N J, Nicholas R J and Walker P J 1991 Semicond. Sci. Technol. 645

[15] Kitamura N, Yamamoto H, Maeda Y, Usami A and Wada T 1987 Semicond. Sci. Technol. 2318

[16] Hidalgo P, Méndez B, Piqueras J, Dutta P S and Dieguez E 1999 Phys. Rev. B 6010613 